\title{
Comparison of the effects of air-powder abrasion, chemical decontamination, or their combination in open-flap surface decontamination of implants failed for peri-implantitis: an ex vivo study
}

\author{
Nicola Pranno ${ }^{1}$ - Maria Paola Cristalli ${ }^{2}$ (D) Fabio Mengoni ${ }^{3} \cdot$ Ilaria Sauzullo $^{3} \cdot$ Susanna Annibali $^{1}$. \\ Antonella Polimeni ${ }^{1}$ • Gerardo La Monaca ${ }^{1}$
}

Received: 2 July 2020 / Accepted: 7 September 2020 / Published online: 25 September 2020

(C) The Author(s) 2020

\begin{abstract}
Objectives To compare, using an ex vivo model, the biofilm removal of three surface decontamination methods following surgical exposure of implants failed for severe peri-implantitis.

Materials and methods The study design was a single-blind, randomized, controlled, ex vivo investigation with intra-subject control. Study participants were 20 consecutive patients with at least 4 hopeless implants, in function for $>12$ months and with progressive bone loss exceeding 50\%, which had to be explanted. Implants of each patient were randomly assigned to the untreated control group or one of the three decontamination procedures: mechanical debridement with air-powder abrasion, chemical decontamination with hydrogen peroxide and chlorhexidine gluconate, or combined mechanical-chemical decontamination. Following surgical exposure, implants selected as control were retrieved, and afterwards, test implants were decontaminated according to allocation and carefully explanted with a removal kit. Microbiological analysis was expressed in colony-forming-units (CFU/ml).

Results A statistically significant difference $(p<0.001)$ in the concentrations of CFU/ml was found between implants treated with mechanical debridement $(531.58 \pm 372.07)$ or combined mechanical-chemical decontamination $(954.05 \pm 2219.31)$ and implants untreated $(37,800.00 \pm 46,837.05)$ or treated with chemical decontamination alone $(29,650.00 \pm 42,596.20)$. No statistically significant difference $(\mathrm{p}=1.000)$ was found between mechanical debridement used alone or supplemented with chemical decontamination. Microbiological analyses identified 21 microbial species, without significant differences between control and treatment groups.

Conclusions Bacterial biofilm removal from infected implant surfaces was significantly superior for mechanical debridement than chemical decontamination.

Clinical relevance The present is the only ex vivo study based on decontamination methods for removing actual and mature biofilm from infected implant surfaces in patients with peri-implantitis.
\end{abstract}

Keywords Air-powder abrasion · Chemical decontamination · Peri-implantitis

Maria Paola Cristalli

mariapaola.cristalli@uniroma1.it

1 Department of Oral and Maxillofacial Sciences, Sapienza University of Rome, Rome, Italy

2 Department of Biotechnologies and Medical Surgical Sciences, Sapienza University of Rome, 6. Caserta St., 00161 Rome, Italy

3 Department of Public Health and Infectious Diseases, Sapienza University of Rome, Rome, Italy

\section{Introduction}

Implant therapy is an effective and predictable method to replace missing teeth with high long-term success and survival rates. Nevertheless, biological complications, i.e., peri-implant mucositis and peri-implantitis, due to the local inflammatory reaction of marginal soft tissues to the biofilm may happen.

Peri-implantitis is an increasing problem, with a wide range of the prevalence, ranging between 9.25 and $12.8 \%$ at the implant level, and between 17 and $22 \%$ at the patient level, due to differences in clinical case definitions [1-3]. 
Peri-implantitis is "a plaque-associated pathological condition, characterized by inflammation in the peri-implant mucosa and subsequent progressive loss of supporting bone" [4].

As peri-implantitis is the effect of an infection process due to the formation of bacterial biofilm on implant surfaces, the target of treatments, either non-surgical or surgical, is to control bacterial infection and peri-implant inflammation. The goal is to stop the disease progression, which can gradually lead to implant loss, and to preserve healthy tissues around functioning implants.

Different strategies in implant surface decontamination, such as mechanical, chemical, photodynamic, and laser therapies, either alone or in various combinations, have been suggested during peri-implant surgery for reducing the bacterial load and removing the biofilm $[5,6]$.

In mechanical debridement, ultrasonic scaler's specific tips, curettes of different materials (stainless steel, titanium, coated carbon fiber, Teflon, or plastic), powdered airabrasive systems, rubber cups, titanium brushes, and abrasive pumice have been used to clean previously contaminated implant surfaces [5-8].

Chemical decontamination consists in topical applications on implant surfaces with saline solution, delmopinol, chlorhexidine, cetylpyridinium chloride (CPC), tetracycline, minocycline, doxycycline, citric acid at $\mathrm{pH} \mathrm{1,} \mathrm{hydrogen} \mathrm{per-}$ oxide, EDTA, or $35 \%$ phosphoric acid gel $[5,6]$.

Photodynamic therapy (PDT) makes use of the diode laser irradiation and photosensitizer solution combination [5, 6, 9]. For laser decontamination, different types of devices, such as Erbium, Chromium: Yttrium, Scandium, Gallium, Garnet (Er,Cr:YSGG), Neodymium-doped Yttrium Aluminum Garnet (Nd:YAG), Erbium: Yttrium Aluminum Garnet ( Er:YAG), Gallium, Aluminum Arsenide ( GaAlAs), carbon dioxide (CO2), are used $[5,6]$.

However, none of these modalities has shown effectiveness in recovering peri-implant health and there is no consensus on the best available treatment to provide satisfactory implant surface decontamination $[5,6]$.

The present study aimed to compare, using an ex vivo model, the efficacy of mechanical debridement with sodium bicarbonate and glycine powders, chemical decontamination with hydrogen peroxide and chlorhexidine gluconate, and combined mechanical-chemical decontamination in the biofilm removal following surgical exposure of implants failed for severe peri-implantitis.

\section{Materials and methods}

\section{Study design}

The study was designed as a single-blind, randomized, controlled, ex vivo investigation with intra-subject control to compare the semiquantitative concentrations of colonyforming units (CFU/ml) and to assess the qualitative microbial composition on the surface of retrieved infected implants clinically treated with three different decontamination methods.

\section{Study population}

Study participants were recruited from patients referred for treatment of peri-implantitis to the Oral Surgery Unit, Policlinico Umberto I, "Sapienza" University of Rome, Italy, between February 2018 and October 2019.

To be included, the patients had to meet the following criteria: (1) at least four osseointegrated implants (unit of statistical analysis) without any restriction about brands, types, and surface, functioning for $>12$ months; (2) progressive bone loss exceeding $50 \%$ of the implant length detected on standard intraoral radiographs; (3) presence of bleeding on gentle probing and/or suppuration. In patients with more than 4 hopeless implants, only 4 with the most severe defect were involved in the study.

The following exclusion criteria were applied: implant mobility, mechanical debridement carried out in the previous 3 months, any peri-implant treatment in the past 6 months, antibiotic therapy during 10 days before surgery.

Each patient received detailed descriptions of the procedure, after which written informed consent was obtained. The protocol was in accordance with the 1975 Declaration of Helsinki on medical protocols and ethics and its later amendments. The study protocol was approved by the Department of Oral and Maxillofacial Sciences-Sapienza, University of Rome, Italy (Protocol identifying number: 0001558).

\section{Randomization}

Before the start of the study, selected implants of each patient were randomly assigned to one of the four groups (untreated control, mechanical debridement, chemical decontamination, mechanical debridement combined with chemical decontamination) using a list of random numbers generated using CLINSTAT software (Martin Bland, York, UK) and sequentially numbered opaque sealed envelopes. The envelopes were opened by the surgeon, and the assigned decontamination method was carried out. The microbiologist assessor was unaware of the delivered treatment. The treatment code was not revealed until all microbiological tests had been completed, and the data file had been established.

\section{Decontamination procedures}

In all groups, treatment was performed by the same surgeon (G.L.M), experienced in the protocol of surface 
decontamination and reconstructive surgery of periimplantitis defects [10].

Immediately prior to intervention, patients rinsed with a $0.2 \%$ chlorhexidine digluconate solution (Corsodyl, GlaxoSmithKline Consumer Healthcare S.p.A. Baranzate, Milan, Italy) for 2 min. Under local anesthesia with $2 \%$ mepivacaine and 1:100,000 adrenalin (Carbocaine, AstraZeneca, Milan, Italy), the prosthetic supra-structure was removed and mucoperiosteal flaps were raised at the buccal and oral aspects. Granulation tissue was removed with titanium curettes (Hufriedy, Chicago, IL, USA) to expose the implant threads and bone defect. Supra- and intrabony implant surfaces were thoroughly irrigated with sterile saline solution for $1 \mathrm{~min}$.

Before decontamination procedures, the implant selected as control was retrieved. Then, each test implant was singularly treated according to allocation and explanted. In order not to influence the results of the other groups, adjacent implants were carefully coated with gauzes soaked with sterile saline solution or, in some cases, postponing overlying soft tissues incision. All implants were explanted using the Implant Retrieval Kit-Nobel Biocare. A specific retrieval instrument mounted through an adapter to the manual torque wrench was put into the implant, which was pulled out with anti-rotational movements and directly transferred in the single tubes, to avoid contamination by bacteria of the oral cavity [11].

At the end of retrieval procedures, mucoperiosteal flaps were repositioned and stabilized with resorbable interrupted sutures (5-0 Vicryl, Ethicon S. p. A. Johnson \& Johnson, Pratica di Mare, Rome, Italy), which were removed after 2 weeks. The postoperative antibiotic protocol included amoxicillin $(875 \mathrm{mg}$ ) plus clavulanic acid (125 mg) (Augmentin, GlaxoSmithKline S.p.A., Verona, Italy) twice daily, and metronidazole (250 mg) (Flagyl, Zambon, Milan, Italy) three times daily for 7 days. Analgesia was achieved with ketoprofen (Ibifen, Istituto Biochimico Italiano G. Lorenzini S. p. A., Aprilia, Latina, Italy) $200 \mathrm{mg}$ for a maximum of three times daily according to individual needs.

\section{Decontamination modalities}

Mechanical debridement was performed with sodium bicarbonate and glycine powders in sequence using the same powered air-abrasion device (PROPHYflex ${ }^{\mathrm{TM}} 3$ with periotip, KaVo, Biberach, Germany) (Fig. 1b, c). The working distance and angulation were individually selected according to the bone defect, and the instrumentation time was for $2 \mathrm{~min}$ per implant $[12,13]$. Chemical decontamination was carried out with cotton pellets soaked with hydrogen peroxide at $3 \%$ for $2 \mathrm{~min}$, followed by $0.2 \%$ chlorhexidine for an additional 1 min (Fig. 1f, g, h). Mechanical debridement supplemented by chemical decontamination was performed in the same ways above mentioned (Fig. 1b, c, e).

\section{Microbiological sampling and analysis}

Each retrieved implant, labeled with a code number, was immediately transferred to the microbiology laboratory into a single tube containing thioglycollate medium and processed via sonication within $6 \mathrm{~h}$ after its removal.

Tubes were vortexed for $30 \mathrm{~s}$ using vortex mixer (VELP Scientifica), sonicated at a frequency of $40 \mathrm{kHz}$ at $22{ }^{\circ} \mathrm{C}$ for 5-7 min (BANDELIN Electronic GmbH \& Co. KG, Berlin, Germany), and then vortexed again for $30 \mathrm{~s}$ to obtain a biofilm disintegration. Sonication fluid was centrifuged (3200 rpm for $15 \mathrm{~min}$ ), the supernatant was aspirated, and the sediment was resuspended in $100 \mu \mathrm{l}$ of medium. A volume of $10 \mu \mathrm{l}$ of medium was placed onto aerobic Columbia sheep blood agar plates and onto anaerobic Schaedler sheep blood agar and incubated for 5 and 10 days, respectively. The semiquantitative estimate was made by counting on plates and expressed in colony-forming units $(\mathrm{CFU} / \mathrm{ml})$. The minimum detection level was $5 \mathrm{CFU} / \mathrm{ml}$. Microbial identification was performed by Bruker MALDI-TOF MS (Bruker Daltonics, Billerica, MA, USA). Species found on plates were identified for each implant.

\section{Sample size calculation}

The effect size value was calculated based on the mean concentrations of $\mathrm{CFU} / \mathrm{ml}$ for each group evaluated in the first five patients (16 implants), using statistics software (GPower 3.1.9.2, Heinrich-Heine-Universität, Düsseldorf, Germany). A power analysis using the repeated measures ANOVA with four measurements, an alpha level of 0.05 , and a medium effect size $(f=0.57)$ showed that 80 implants would be adequate to obtain $95 \%$ power in detecting a statistical difference in the $\mathrm{CFU} / \mathrm{ml}$ between control and treatment groups assuming a loss of $20 \%$ of the sample during all procedures [14].

\section{Statistical analysis}

The implant was chosen as the unit for the statistical analysis.

Data were evaluated using standard statistical analysis software (version 20.0, Statistical Package for the Social Sciences, IBM Corporation, Armonk, NY, USA). A database was created using Excel (Microsoft, Redmond, WA, USA). Descriptive statistics including mean \pm SD values and percentage were calculated for each variable: concentrations of CFU/ $\mathrm{ml}$ and microbiological differences.

The Shapiro-Wilk test was used to determine whether or not the data conformed to a normal distribution. As a nonparametric distribution of the concentrations of $\mathrm{CFU} / \mathrm{ml}$ between four groups was found, a Kruskal-Wallis test was conducted to determine differences in the implant surface detoxification treatments. Pairwise comparisons were performed using Dunn's procedure [15] with a Bonferroni correction 
Fig. 1 Implant surface decontamination: a the mucoperiosteal flap raised; $\mathbf{b}$ sodium bicarbonate powderbased air abrasion; $\mathbf{c}$ glycine powder-based air abrasion; $\mathbf{d}$ retrieval of the mechanically decontaminated test implant; $\mathbf{e}$ chemical decontamination of the remaining implant performed after mechanical debridement; $\mathbf{f}$ implants before chemical decontamination; $\mathbf{g}$ hydrogen peroxide at $3 \%$; $\mathbf{h}$ chlorhexidine gluconate at $2 \%$

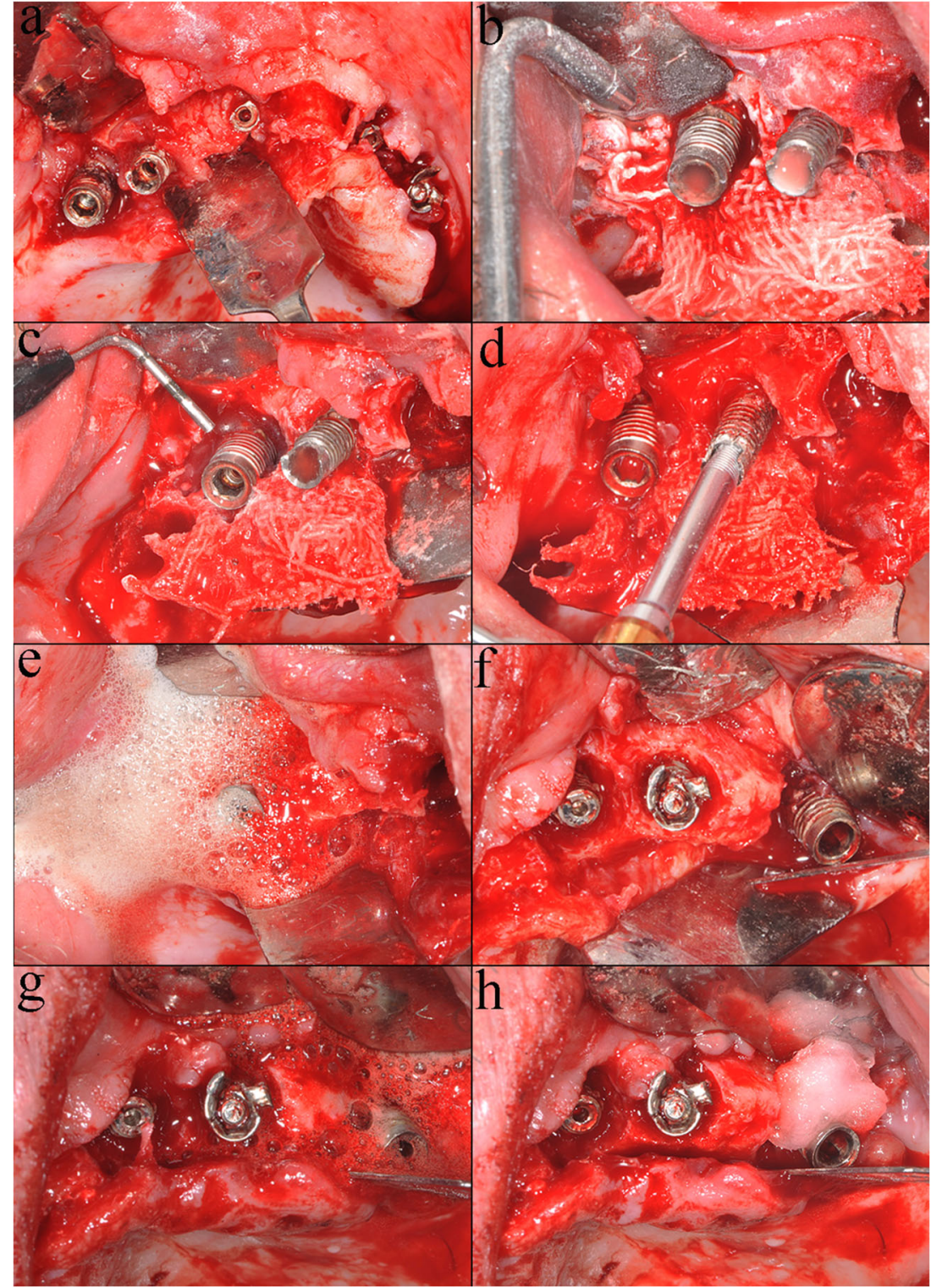

for multiple comparisons. Adjusted $p$ values are presented. Fisher's exact test was used to evaluate the presence of qualitative microbiological difference. In each test, the cut-off for statistical significance was $p \leq 0.05$.

\section{Results}

Twenty consecutive patients (11 males and 9 females; age $65.75 \pm 9.67$ years) aged $>18$ years were selected for a total of 80 implants with different rough surfaces (4 for each subject). No test or control implants were lost during decontamination procedures or the incubation period. In all groups, no intraoperative complications occurred (e.g., emphysema formation) and the postoperative wound healing was uneventful.

\section{Semiquantitative microbiological analysis}

The semiquantitative microbiological analysis found mean values of 37,800.00 $\pm 46,837.05 \mathrm{CFU} / \mathrm{ml}$ for untreated implants, $531.58 \pm 372.07 \mathrm{CFU} / \mathrm{ml}$ for implants treated with mechanical debridement, $29,650.00 \pm 42,596.20$ for implants treated with chemical decontamination, and $954.05 \pm 2219.31$ for implants treated with combined mechanical-chemical decontamination.

The concentrations of CFU/ml for each group were presented in a bar chart illustrating a lower value in mean counts only in the group treated with mechanical debridement and with mechanical debridement combined with chemical decontamination (Fig. 2).

Differences in the concentrations of $\mathrm{CFU} / \mathrm{ml}$ were statistically significant $(p<0.001)$ when mechanical debridement alone or supplemented by chemical decontamination was compared with 
Fig. 2 Bar chart based on the $\log 10$ of mean CFU. The error bar represented the standard deviation of mean $\mathrm{CFU} / \mathrm{ml}$ expressed using logarithmic notation. The graph showed a major number of CFU in the control group and in the group treated with chemical agents compared with both groups treated with mechanical debridement and with mechanical debridement combined with chemical decontamination.

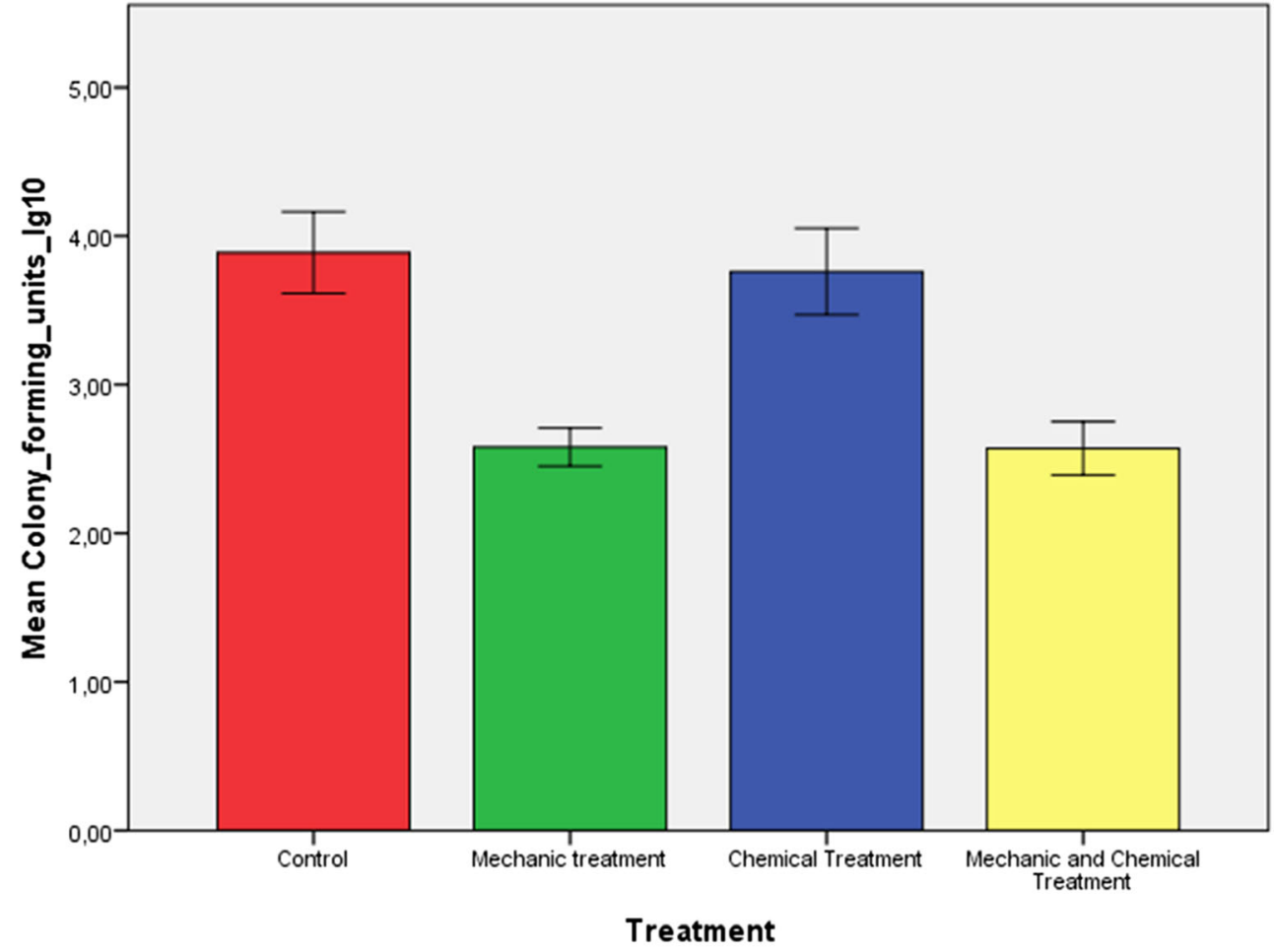

Error Bars: $95 \% \mathrm{Cl}$ implants untreated (positive control group) or treated with chemical decontamination (Fig. 3). Conversely, no statistically significant difference ( $p=1.000$ ) was found comparing chemical decontamination to untreated implants and mechanical debridement to combined mechanical-chemical decontamination (Fig. 3).

\section{Microbiological findings}

The microbiological analysis of 80 implants affected by periimplantitis identified 21 microbial species. The most frequent bacteria were Staphylococcus aureus (40 implants), Streptococcus mitis/oralis (32 implants), and Staphylococcus epidermidis and Streptococcus salivarius (20 implants). Enterococcus faecalis and Candida albicans were found in 16 and 12 implants, respectively, and Pseudomonas aeruginosa and Neisseria flavescens in 8 implants. The frequency of microorganisms detected in control and test implants is reported in Table 1. There was no statistically significant difference in the microbial species between control and treatment groups as assessed by Fisher's exact test ( $p=0.917)$. In short, implants affected by peri-implantitis, regardless of their site in the oral cavity, showed the same microbiota.

\section{Discussion}

The results of the microbiologic analysis revealed that implants treated during open-flap surgery with mechanical debridement or with mechanical debridement combined with chemical decontamination presented a statistically significant difference in the concentrations of $\mathrm{CFU} / \mathrm{ml}$ when comparing to implants untreated (positive control group) or treated with chemical decontamination alone.

A direct comparison of these findings to those previously reported in the literature is difficult because to the best of the authors' knowledge, no ex vivo studies comparing the efficacy of decontamination methods on the removal of microorganisms from implants surfaces in peri-implantitis defects were available.

In the present study, the choice of sodium bicarbonate and amino-acid glycine air powders for mechanical debridement was due to effectiveness in cleaning contaminated implant surfaces, mainly in the deepest part of peri-implant defects, which is difficult to achieve [16-21]. Air powder abrasive treatments have proven effective in removing biofilm (range 85-100\%), and in improving marginal bleeding, bleeding on probing, suppuration, and probing depth in open surgical procedures [7, 22-25]. In in vitro studies, sodium bicarbonate showed to remove more than $84 \%$ of bacteria or bacterial products, even if it produced slight to medium surface changes, such as small crater-like defects, rounding, or removal of sharp edges [7]. Amino-acid glycine was also able to remove single bacterial species and plaque from smooth and structured titanium surfaces, and it was less abrasive than sodium bicarbonate powder [7, 12, 26, 27]. The lower momentum and less energy at impact onto implant surface were due to the 
Fig. 3 Pairwise comparison of decontamination methods. In the diagram, the numbers and the joining lines reflected respectively the average rank and the pairwise comparison for each group. Orange and black lines reflected pairwise comparisons, respectively statistically significant and not significant. The table below the diagram showed pairwise comparisons in more detail: the first column indicated which pairwise comparison was made and in what direction; the second column reported the test statistic (difference between mean ranks of the two groups); the third and fourth columns presented the standard error and the standardized statistic test; the fifth and the sixth columns showed the unadjusted and adjusted $p$ value.

\section{Pairwise Comparisons of Treatment}

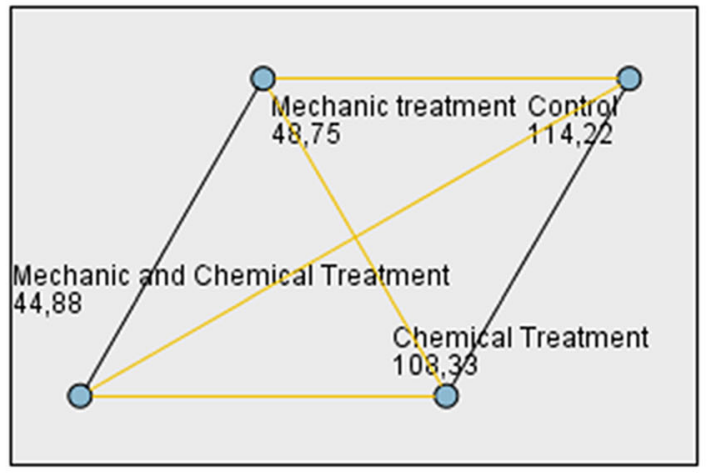

Each node shows the sample average rank of Treatment.

\begin{tabular}{|c|c|c|c|c|c|}
\hline Sample1-Sample2 & $\begin{array}{c}\text { Test } \\
\text { Statistic }\end{array}$ & $\begin{array}{l}\text { Std. } \\
\text { Error }\end{array}$ & $\begin{array}{l}\text { Std. Test } \\
\text { Statistic }\end{array}$ & Sig. & Adj.Sig. \\
\hline $\begin{array}{l}\text { Mechanic and Chemical } \\
\text { Treatment-Mechanic treatment }\end{array}$ & 3,872 & 10,525 &, 368 & ,713 & 1,000 \\
\hline $\begin{array}{l}\text { Mechanic and Chemical } \\
\text { Treatment-Chemical Treatment }\end{array}$ & 63,455 & 10,275 & 6,176 &, 000 &, 000 \\
\hline $\begin{array}{l}\text { Mechanic and Chemical } \\
\text { Treatment-Control }\end{array}$ & 69,344 & 10,113 & 6,857 &, 000 &, 000 \\
\hline $\begin{array}{l}\text { Mechanic treatment-Chemical } \\
\text { Treatment }\end{array}$ & $-59,583$ & 10,203 & $-5,840$ &, 000 &, 000 \\
\hline Mechanic treatment-Control & 65,472 & 10,040 & 6,521 &, 000 &, 000 \\
\hline Chemical Treatment-Control & 5,889 & 9,777 & 602 &, 547 & 1,000 \\
\hline
\end{tabular}

Each row tests the null hypothesis that the Sample 1 and Sample 2 distributions are the same.

Asymptotic significances (2-sided tests) are displayed. The significance level is, 05 . lower density and hardness and the smaller size of the particles [18]. Sodium bicarbonate larger-sized particles proved to have mechanical removal and impact on implant roughness more significant than glycine small-sized particles [18, 28]. Furthermore, also the greater efficacy in restoring biocompatibility of sodium bicarbonate compared with glycine was probably due to the higher ablation power of its harder and larger particles in the removal of the carbon layer produced by the activity of the biofilm [24].

The different ability of the two powder formulations in removing a biofilm was the rationale at the basis of the sequential use of sodium bicarbonate and glycine [18, 28]. Using small after larger powder particles should be helpful to reach the more difficult areas to clean in the rough implants and to increase surface abrasion because the higher solubility potential reduces the presence of undissolved particles in the water-air stream [28].

Chemical decontamination with hydrogen peroxide plus chlorhexidine gluconate was adopted as these are the most common and extensively antiseptic agents used in periodontics. Furthermore, their association should enhance bactericidal effects, exploiting the synergy between the two different mechanisms of action: the oxidizing effect of hydrogen peroxide and the disruption of the bacterial cell membrane of chlorhexidine gluconate [10].

The limited efficacy of chemical decontamination on infected implant surfaces found in the present investigation was confirmed by the results of other studies [29-31].

Reduced efficacy of the detoxification with hydrogen peroxide 3\% was reported in in vitro studies. Zablotsky et al. [32] showed that burnishing with cotton pellets soaked by hydrogen peroxide $3 \%$ for $1 \mathrm{~min}$ removed significantly more endotoxin from grit-blasted titanium alloy strips contaminated with Escherichia coli lipopolysaccharide than untreated controls, but not compared with specimens treated with sterile saline alone. Mouhyi et al. [33] found that hydrogen peroxide at low concentrations was not effective in removing contaminants 
Table 1 Frequency of microorganisms detected in control and test implants

\begin{tabular}{lllll}
\hline Types of bacteria & Frequency (implants) & Percent & Valid percent & Cumulative percent \\
\hline Micrococcus luteus & 8 & 3.0 & 3.0 & 3.0 \\
Strep. constellatus & 8 & 3.0 & 3.0 & 6.1 \\
Strep. mitis/oralis & 32 & 12.1 & 12.1 & 18.2 \\
Candida albicans & 12 & 4.5 & 4.5 & 22.7 \\
Strep. salivarius & 20 & 7.6 & 7.6 & 30.3 \\
Neisseria subflave & 12 & 4.5 & 4.5 & 34.8 \\
Staf. epidermidis & 24 & 9.1 & 9.1 & 43.9 \\
Enterococcus faecalis & 16 & 6.1 & 6.1 & 50.0 \\
Staf. aureus & 40 & 15.2 & 15.2 & 65.2 \\
Pseudomonas aeruginosa & 8 & 3.0 & 3.0 & 68.2 \\
Neisseria flavescens & 8 & 3.0 & 3.0 & 71.2 \\
Strep. parasanguinis & 20 & 7.6 & 7.6 & 78.8 \\
Strep. pneumoniae & 8 & 3.0 & 3.0 & 81.8 \\
Strep. vestibularis & 8 & 3.0 & 3.0 & 84.8 \\
Strep. epidermidis & 8 & 3.0 & 3.0 & 87.9 \\
Strep. viridans & 8 & 3.0 & 3.0 & 90.9 \\
Klebsiella oxytoca & 4 & 1.5 & 1.5 & 92.4 \\
Eikella corrodens & 4 & 1.5 & 1.5 & 93.9 \\
Lactobacillus paracasei & 8 & 3.0 & 3.0 & 97.0 \\
Lactobacillus rhamnosus & 4 & 1.5 & 1.5 & 98.5 \\
Veillonella parvula & 4 & 1.5 & 1.5 & 100.0 \\
Total & 264 & 100.0 & 100.0 & \\
\hline
\end{tabular}

from commercially pure titanium foils placed on dentures in volunteer patients simulating a peri-implantitis situation. Bürgers et al. [34] assessing the effect of various topical antiseptics on plane titanium specimens contaminated by Staphylococcus epidermidis, Candida albicans, or Streptococcus sanguinis, demonstrated that hydrogen peroxide $3 \%$ was solely effective against Candida albicans.

Limited effects in decontamination of implants surface were also reported for chlorhexidine. In two randomized, double-blind, controlled trials, treatment of implant surfaces with chlorhexidine $0.12 \%$ + cetylpyridinium chloride $0.05 \%$ or chlorhexidine $2.0 \%$ during resective surgery for periimplantitis led to a significant reduction of anaerobic bacteria compared with placebo, even if the decrease of bacterial load did not turn into better clinical or radiographic treatment outcomes over 12 months $[31,35]$. In an in vivo study on the antimicrobial effectiveness of six different antiseptic solutions on machined titanium specimens exposed overnight in the oral cavity of four volunteers, chlorhexidine demonstrated some efficacy in reducing the bacterial load and significant bactericidal effects against adhering bacteria [29]. The explanation of limited effects of chlorhexidine might be researched both in the brief contact time with implants surface and in copious irrigations with sterile saline solution after use. This situation could interfere with the antimicrobial effects of chlorhexidine in peri-implant lesions, diminishing its binding ability with hard and soft tissue due to the slow-release (substantivity property) [31].

In the present study, the adjunct of antiseptics to mechanical debridement, although proposed in the literature for the treatment of peri-implant infection, showed no statistically significant difference in mean counts of CFU compared with mechanical debridement alone. The data was corroborated by the results of Porras et al. [36], who did not find additional improvements in non-surgical therapy of mucositis when mechanical cleansing and oral hygiene instructions were supplemented by the local irrigation with chlorhexidine $0.12 \%$ and topical application of a $0.12 \%$ chlorhexidine gel. In a prospective randomized controlled clinical trial on the surgical treatment of severe peri-implantitis, the local application of chlorhexidine $0.2 \%$ after the removal of the hard deposits with titanium-coated curettes had no overall impact on clinical and radiological outcomes [37].

Microbiota detected in the present investigation was almost the same at both positive controls and treated implants, regardless of the site in the oral cavity, demonstrating that the removal of bacterial biofilm from the infected implant surfaces is quantitative and not qualitative. The highest prevalence of Staphylococcus aureus was consistent with microbiological results at implant sites with varying degrees of inflammation reported in other studies [38-41], and was justified by its high affinity for titanium surfaces [42-44]. The other bacteria 
found in the present investigation were comparable with those reported in some observational studies, in which periimplantitis was considered a complex and heterogenous infection, more frequently linked with opportunistic pathogens not primarily associated with periodontitis, such as Staphylococci, Pseudomonas aeruginosa, Enterics, and Candida species [45-51]. Indeed, most Staphylococci can become from commensal bacteria to pathogens in the presence of implanted medical devices $[52,53]$. Furthermore, different species of the genus Staphylococcus can colonize implant surfaces affected by peri-implantitis with a prevalence higher than periodontal pathogenic bacteria such as Tannerella forsythia and Porphyromonas gingivalis [38-40,54]. Unexpectedly, microbiologic assays did not identify several species of common periodontal pathogenic bacteria.

Strengths of the present ex vivo study were to overcome some limitations of in vitro and animal studies as well as in human clinical trials.

In in vitro studies, specimens (titanium discs, sheets, strips and cylinders) and biofilm contaminants (non-mineralized supragingival plaque, single-species biofilm, bacterial products, indelible non-covering ink) could not represent actual clinical situations, due to differences in the macrostructure (threads shape) and qualitative and quantitative composition of plaque of contaminated implants [7, 12, 20,21, 27, 29]. Additionally, also the use of custom-made defect models with different morphologies mimicking peri-implant defects was unable to simulate clinical settings, in which treatment outcomes were influenced by many factors such as patient's characteristics or the presence of the suprastructure [7, 8]. Furthermore, cleaning efficacy of decontamination procedures was different in in vivo or in vitro models, as the anatomical limitations of the oral cavity (e.g., the tongue) and the presence of blood and saliva hamper the accessibility to infected implant surfaces.

In animal studies, the main limitation was the difficulty to directly transfer therapeutic effects on peri-implant infection, which were on average better in animal models (mainly monkeys and dogs) than humans, due to the difference in anatomical characteristics and physiological systems between the two species $[55,56]$.

In human clinical trials, treatment outcomes are significantly influenced by patient's factors, such as the level of oral hygiene, peri-implant microbiota, prosthetic designs, immunocompetent characteristics, systemic conditions, history of periodontitis, and cigarette smoking [5]. Further confounder factors were the absence of a true control group (untreated patients) for ethical reasons, the allocation of different types of implants in test and control groups, and the use of clinical parameters instead of the quantification of the residual biofilm as treatment outcomes $[30,57]$.

Strength points of the present study were the use of failed contaminated implants and not patients as statistical analysis unit, decontamination methods applied before the implants retrieval, untreated implants used as positive control tests, the intra-subject evaluation adopted to overcome the bias on treatment effects due to the implant design and surface, and patient's factors.

Nevertheless, several limitations were present. The main was the wide range of rough surface implant topographies, which may have influenced the microbial adhesion and complex biofilm formation, and the effectiveness of decontamination methods. Further limitations were the lack of investigation on changes of chemical and physical properties in implant surfaces after instrumentations and the semiquantitative analysis of the peri-implantitis microbiota, instead of cultureindependent techniques.

Given increasing worldwide use of dental implants and inevitable augmentation of peri-implantitis cases, additional researches on the decontamination/detoxification of infected titanium surfaces are needed to identify an effective treatment.

\section{Conclusion}

Within above-mentioned limits, in the present ex vivo study, the removal of bacterial biofilm from infected implant surfaces was significantly superior for mechanical debridement with sodium bicarbonate and glycine powders than chemical decontamination with hydrogen peroxide and chlorhexidine gluconate. However, these results must be interpreted with caution, as no decontamination procedure has shown to achieve complete elimination of the biofilm.

Acknowledgments The authors wish to thank Gianluca Puggioni for his help in microbiological analysis.

Author contributions Polimeni A and Annibali S contributed to conception and design of the study, data analysis, and interpretation, and critically revised the manuscript; Cristalli MP contributed to data acquisition, analysis and interpretation, and drafted the manuscript; La Monaca G contributed to data acquisition, analysis and interpretation, and critically revised the manuscript; Pranno N contributed to statistics, data analysis and interpretation, and drafted the manuscript; Mengoni F and Sauzullo I contributed to antimicrobial analysis, data acquisition, analysis and interpretation, and drafted the manuscript. All authors gave final approval and agree to be accountable for all aspects of the work.

Funding Open access funding provided by Università degli Studi di Roma La Sapienza within the CRUI-CARE Agreement.

\section{Compliance with ethical standards}

Conflict of interest The authors declare that they have no conflict of interest.

Ethical approval All procedures performed in the study were in accordance with the 1964 Declaration of Helsinki on medical protocols and ethics and its later amendments and approved by the Department of Oral and Maxillofacial Sciences-Sapienza, University of Rome, Italy (Protocol identifying number: 0001558). 
Informed consent Informed consent was obtained from all individual participants included in the study.

Open Access This article is licensed under a Creative Commons Attribution 4.0 International License, which permits use, sharing, adaptation, distribution and reproduction in any medium or format, as long as you give appropriate credit to the original author(s) and the source, provide a link to the Creative Commons licence, and indicate if changes were made. The images or other third party material in this article are included in the article's Creative Commons licence, unless indicated otherwise in a credit line to the material. If material is not included in the article's Creative Commons licence and your intended use is not permitted by statutory regulation or exceeds the permitted use, you will need to obtain permission directly from the copyright holder. To view a copy of this licence, visit http://creativecommons.org/licenses/by/4.0/.

\section{References}

1. Rakic M, Galindo-Moreno P, Monje A, Radovanovic S, Wang HL, Cochran D, Sculean A, Canullo L (2018) How frequent does periimplantitis occur? A systematic review and meta-analysis. Clin Oral Investig 22(4): 1805-1816

2. Muñoz V, Duque A, Giraldo A, Manrique R (2018) Prevalence of peri-implant disease according to periodontal probing depth and bleeding on probing: a systematic review and meta-analysis. Int $\mathrm{J}$ Oral Maxillofac Implants 33(4):e89-e105

3. Cosgarea R, Sculean A, Shibli JA, Salvi GE (2019) Prevalence of peri-implant diseases - a critical review on the current evidence. Braz Oral Res 33(suppl 1):e063

4. Berglundh T, Armitage G, Araujo MG et al (2018) Peri-implant diseases and conditions: Consensus report of workgroup 4 of the 2017 World Workshop on the Classification of Periodontal and Peri-Implant Diseases and Conditions. J Periodontol 89(Suppl 1): 313-318

5. Figuero E, Graziani F, Sanz I, Herrera D, Sanz M (2014) Management of peri-implant mucositis and peri-implantitis. Periodontol 66(1):255-273

6. Renvert S, Polyzois IN (2015) Clinical approaches to treat periimplant mucositis and peri-implantitis. Periodontol 68(1):369-404

7. Louropoulou A, Slot DE, Van der Weijden F (2014) The effects of mechanical instruments on contaminated titanium dental implant surfaces: a systematic review. Clin Oral Implants Res 25(10): $1149-1160$

8. Tastepe CS, Lin X, Werner A, Donnet M, Wismeijer D, Liu Y (2018) Cleaning effect of osteoconductive powder abrasive treatment on explanted human implants and biofilm-coated titanium discs. Clin Exp Dent Res 4(1):25-34

9. Marotti J, Tortamano P, Cai S, Ribeiro MS, Franco JE, de Campos TT (2013) Decontamination of dental implant surfaces by means of photodynamic therapy. Lasers Med Sci 28(1):303-309

10. La Monaca G, Pranno N, Annibali S, Cristalli MP, Polimeni A (2018) Clinical and radiographic outcomes of a surgical reconstructive approach in the treatment of peri-implantitis lesions: a 5-year prospective case series. Clin Oral Implants Res 29(10):1025-1037

11. Roy M, Loutan L, Garavaglia G, Hashim D (2020) Removal of osseointegrated dental implants: a systematic review of explantation techniques. Clin Oral Investig 24(1):47-60

12. Sahrmann P, Ronay V, Sener B, Jung RE, Attin T, Schmidlin PR (2013) Cleaning potential of glycine air-flow application in an in vitro peri-implantitis model. Clin Oral Implants Res 24:666-670

13. Tastepe CS, Lin X, Donnet M, Wismeijer D, Liu Y (2017) Parameters that improve cleaning efficiency of subgingival air polishing on titanium implant surfaces: an in vitro study. $\mathrm{J}$ Periodontol 88(4):407-414

14. Faul F, Erdfelder E, Lang A, Buchner A (2007) G*Power 3: a flexible statistical power analysis program for the social, behavioral, and biomedical sciences. Behav Res Methods 39:175-191

15. Dunn OJ (1964) Multiple comparisons using rank sums. Technometrics 6:241-252

16. Schwarz F, Bieling K, Bonsmann M, Latz T, Becker J (2006) Nonsurgical treatment of moderate and advanced periimplantitis lesions: a controlled clinical study. Clin Oral Investig 10(4):279288

17. John G, Becker J, Schwarz F (2016) Effectivity of air-abrasive powder based on glycine and tricalcium phosphate in removal of initial biofilm on titanium and zirconium oxide surfaces in an ex vivo model. Clin Oral Investig 20(4):711-719

18. Wei MCT, Tran C, Meredith N, Walsh LJ (2017) Effectiveness of implant surface debridement using particle beams at differing air pressures. Clin Exp Dent Res 3(4):148-153

19. Quintero DG, Taylor RB, Miller MB, Merchant KR, Pasieta SA (2017) Air-abrasive disinfection of implant surfaces in a simulated model of periimplantitis. Implant Dent 26(3):423-428

20. Keim D, Nickles K, Dannewitz B, Ratka C, Eickholz P, Petsos H (2019) In vitro efficacy of three different implant surface decontamination methods in three different defect configurations. Clin Oral Implants Res 30(6):550-558

21. Steiger-Ronay V, Merlini A, Wiedemeier DB, Schmidlin PR, Attin T, Sahrmann P (2017) Location of unaccessible implant surface areas during debridement in simulated peri-implantitis therapy. BMC Oral Health 17:137

22. Tastepe CS, van Waas R, Liu Y, Wismeijer D (2012) Air powder abrasive treatment as an implant surface cleaning method: a literature review. Int J Oral Maxillofac Implants 27(6):1461-1473

23. Louropoulou A, Slot DE, Van der Weijden F (2015) Influence of mechanical instruments on the biocompatibility of titanium dental implants surfaces: a systematic review. Clin Oral Implants Res 26(7):841-850

24. Moharrami M, Perrotti V, Iaculli F, Love RM, Quaranta (2019) A. Effects of air abrasive decontamination on titanium surfaces: a systematic review of in vitro studies. Clin Implant Dent Relat Res 21(2):398-421

25. Lasserre JF, Brecx MC, Toma S (2020) Implantoplasty versus glycine air abrasion for the surgical treatment of peri-implantitis: a randomized clinical trial. Int J Oral Maxillofac Implants 35(35): 197-206

26. Ronay V, Merlini A, Attin T, Schmidlin PR, Sahrmann P (2017) In vitro cleaning potential of three implant debridement methods. Simulation of the non-surgical approach. Clin Oral Implants Res 28:151-155

27. Sahrmann P, Ronay V, Hofer D, Attin T, Jung RE, Schmidlin PR (2015) In vitro cleaning potential of three different implant debridement methods. Clin Oral Implants Res 26:314-319

28. Matsubara VH, Leong BW, Leong MJL, Lawrence Z, Becker T, Quaranta A (2020) Cleaning potential of different air abrasive powders and their impact on implant surface roughness. Clin Implant Dent Relat Res 22(1):96-104

29. Gosau M, Hahnel S, Schwarz F, Gerlach T, Reichert TE, Bürgers R (2010) Effect of six different peri-implantitis disinfection methods on in vivo human oral biofilm. Clin Oral Implants Res 21(8):866872

30. Ntrouka VI, Slot DE, Louropoulou A, Van der Weijden F (2011) The effect of chemotherapeutic agents on contaminated titanium surfaces: a systematic review. Clinl Oral Implants Res 22(7):681690

31. de Waal YC, Raghoebar GM, Huddleston Slater JJ, Meijer HJ, Winkel EG, van Winkelhoff AJ (2013) Implant decontamination 
during surgical peri-implantitis treatment: a randomized, doubleblind, placebo-controlled trial. J Clin Periodontol 40(2):186-195

32. Zablotsky MH, Diedrich DL, Meffert RM (1992) Detoxification of endotoxin-contaminated titanium and hydroxyapatite-coated surfaces utilizing various chemotherapeutic and mechanical modalities. Implant Dent 1(2):154-158

33. Mouhyi J, Sennerby L, Wennerberg A, Louette P, Dourov N, van Reck J (2000) Re-establishment of the atomic composition and the oxide structure of contaminated titanium surfaces by means of carbon dioxide laser and hydrogen peroxide: an in vitro study. Clin Implant Dent Relat Res 2(4):190-202

34. Bürgers R, Witecy C, Hahnel S, Gosau M (2012) The effect of various topical peri-implantitis antiseptics on Staphylococcus epidermidis, Candida albicans, and Streptococcus sanguinis. Arch Oral Biol 57(7):940-947

35. de Waal YC, Raghoebar GM, Meijer HJ, Winkel EG, van Winkelhoff AJ (2015) Implant decontamination with $2 \%$ chlorhex-idine during surgical peri-implantitis treatment: a randomized, dou-ble-blind, controlled trial. Clin Oral Implants Res 26: $1015-1023$

36. Porras R, Anderson GB, Caffesse R, Narendran S, Trejo PM (2002) Clinical response to 2 different therapeutic regimens to treat periimplant mucositis. J Periodontol 73(10):1118-1125

37. Carcuac O, Derks J, Charalampakis G, Abrahamsson I, Wennström J, Berglundh T (2016) Adjunctive systemic and local antimicrobial therapy in the surgical treatment of peri-implantitis: a randomized controlled clinical trial. J Dent Res 95:50-57

38. Persson GR, Samuelsson E, Lindahl C, Renvert S (2010) Mechanical non-surgical treatment of peri-implantitis: a singleblinded randomized longitudinal clinical study. II. Microbiological results. J Clin Periodontol 37(69):563-573

39. Persson GR, Roos-Jansåker AM, Lindahl C, Renvert S (2011) Microbiologic results after non-surgical erbium-doped:yttrium, aluminum, and garnet laser or air-abrasive treatment of periimplantitis: a randomized clinical trial. J Periodontol 82(9):12671278

40. Persson GR, Renvert S (2016) Cluster of bacteria associated with peri-implantitis. J Periodontal Res 51(6):689-698

41. Marsh PD, Zaura E (2017) Dental biofilm: ecological interactions in health and disease. J Clin Periodontol 44(Suppl. 18):S12-S22. https://doi.org/10.1111/jepe.12679

42. Fürst MM, Salvi GE, Lang NP, Persson GR (2006) Bacterial colonization immediately after installation on oral titanium implants. Clin Oral Implants Res 18:501-508

43. Harris LG, Mead L, Müller-Oberländer E, Richards RG (2006) Bacteria and cell cytocompatibility studies on coated medical grade titanium surfaces. J Biomed Mater Res A 78(1):50-58
44. Harris LG, Meredith DO, Eschbach L, Richards RG (2007) Staphylococcus aureus adhesion to standard micro-rough and electropolished implant materials. J Mater Sci Mater Med 18(6): 1151-1156

45. Leonhardt A, Renvert S, Dahlen G (1999) Microbial findings at failing implants. Clin Oral Implants Res 10:339-345

46. Mombelli A, Decaillet F (2011) The characteristics of biofilms in peri-implant disease. J Clin Periodontol 38(Suppl 11):203-213

47. Schwarz F, Becker K, Rahn S, Hegewald A, Pfeffer K, Henrich B (2015) Realtime PCR analysis of fungal organisms and bacterial species at periimplantitis sites. Int J Implant Dent 1(1):9

48. Albertini M, Lopez-Cerero L, O’Sullivan MG et al (2015) Assessment of periodontal and opportunistic flora in patients with peri-implantitis. Clin Oral Implants Res 26:937-941

49. Rakic M, Grusovin MG, Canullo L (2016) The microbiologic profile associated with peri-implantitis in humans: a systematic review. Int J Oral Maxillofac Implants 31(2):359-368

50. Padial-Molina M, Lopez-Martinez J, O'Valle F, Galindo-Moreno P (2016) Microbial profiles and detection techniques in peri-implant diseases: a systematic review. J Oral Maxillofac Res 7(3):e10

51. Schwarz F, Derks J, Monje A, Wang HL (2018) Peri-implantitis. J Periodontol 89(Suppl 1):S267-S290

52. Cook A, Berne J, Norwood S (2009) The incidence and complications of methicillinresistant Staphylococcus aureus in a community Level I trauma center. J Trauma 67:102-107

53. Fujimura S, Sato T, Kikuchi T, Zaini J, Gomi K, Watanabe A (2009) Efficacy of clarithromycin plus vancomycin in mice with implant-related infection caused by biofilm forming Staphylococcus aureus. J Orthop Sci 14:658-661

54. Sabogal MA, Castillo DM et al (2017) Microbiome and microbial biofilm profiles of peri-implantitis: a systematic review. J Periodontol 88(10):1066-1089

55. Faggion CM Jr, Chambrone L, Gondim V, Schmitter M, Tu YK (2010) Comparison of the effects of treatment of peri-implant infection in animal and human studies: systematic review and metaanalysis. Clin Oral Implants Res 21(2):137-147

56. Schwarz F, Iglhaut G, Becker J (2012) Quality assessment of reporting of animal studies on pathogenesis and treatment of periimplant mucositis and peri-implantitis. A systematic review using the ARRIVE guidelines. J Clin Periodontol 39(Suppl. 12):63-72

57. Graziani F, Figuero E, Herrera D (2012) Systematic review of quality of reporting, outcome measurements and methods to study efficacy of preventive and therapeutic approaches to peri-implant diseases. J Clin Periodontol 39(Suppl 12):224-244

Publisher's note Springer Nature remains neutral with regard to jurisdictional claims in published maps and institutional affiliations. 